Check for updates

Cite this: RSC Adv., 2018, 8, 26133

\title{
Ultrafast nonlinear optical properties and carrier dynamics of silver nanoparticle-decorated $\mathrm{ZnO}$ nanowires
}

\begin{abstract}
Ke-Xin Zhang, Cheng-Bao Yao, (D)* Xing Wen, Qiang-Hua Li and Wen-Jun Sun
Silver (Ag) nanoparticle-decorated zinc oxide ( $\mathrm{ZnO}$ ) nanowires ( $\mathrm{Ag}-\mathrm{ZnO}$ ) have been successfully synthesized by chemical vapour deposition and the magnetron sputtering method. Scanning electron microscopy images indicate that Ag nanoparticles are distributed uniformly on the surface of the $\mathrm{ZnO}$ nanowires. The results of room temperature photoluminescence (RTPL) reveal two major emission peaks for the $\mathrm{Ag}-\mathrm{ZnO}$ nanowires, and the emission peaks in the visible region are stronger than those of the unmodified $\mathrm{ZnO}$ nanowires. The mechanism of RTPL and low temperature photoluminescence (LTPL) emission is discussed in detail. Nonlinear optical properties and ultrafast dynamics have been investigated using the Z-scan and two color pump-probe (TCPP) techniques, respectively. The nonlinear absorption properties in the nano-, pico- and femto-second regime have been analyzed using the singlet state three-level and four-level models, respectively. The samples show self-focusing nonlinearity and good two-photon absorption (TPA)-induced ground state saturation absorption as well as excited state reverse saturable absorption behavior. For the nanosecond and picosecond pulses, the reverse saturated absorption in the excited state mainly originates from the absorption at low excited states or deep levels; however, for the femtosecond pulse, it is caused by the absorption at high excited states. The TCPP results show that the ground state or deep level light bleaching (for nano- and pico-second regime) and TPA-induced excited-state absorption (for femtosecond regime) behaviors range from $470 \mathrm{~nm}$ to $620 \mathrm{~nm}$. The remarkable nonlinear optical properties reveal that $\mathrm{Ag}-\mathrm{ZnO}$ nanowires are potential nanocomposite materials for the development of nonlinear optical devices.
\end{abstract}

Received 9th April 2018

Accepted 29th June 2018

DOI: 10.1039/c8ra03027h

rsc.li/rsc-advances
In recent years, a number of ultrafast optical techniques have been employed to investigate ultrafast nonlinear optical and carrier dynamics of organic and inorganic semiconductors; ${ }^{15-19}$ a variety of new metal and semiconductor nanocomposites have also been reported recently. ${ }^{20-22}$ Among them, the $\mathrm{Ag}$. nanoparticle-decorated $\mathrm{ZnO}(\mathrm{Ag}-\mathrm{ZnO})$ nanostructures have been synthesized by various methods and technologies such as the low-temperature hydrothermal method, ${ }^{23}$ hydrothermal and chemical method, ${ }^{24}$ wet chemical synthesis and thermal evaporation process. ${ }^{25}$ The surface plasmon resonance (SPR) effect of a noble metal can expand the response range of incident light, increase optical absorption, enrich the electrode with light electrons increasing the light transmission rate, prolong the life of electronic devices and inhibit photoproduction of electron-holes in the composites. ${ }^{26-28}$ By decorating oxide photoanodes with $\mathrm{Ag}$, the absorption of visible light is enhanced and the combination of photogenerated electron-holes is suppressed; however, the technological process is highly complex and requires high cost and also, large-scale applications are not possible because of vacuum-proof binders for printing technologies. Furthermore, to date, nonlinear optical properties and carrier dynamics of the Ag-ZnO nanowires have not been reported. In the present paper, the $\mathrm{Ag}-\mathrm{ZnO}$ nanowire composites
Key Laboratory of Photonic and Electric Bandgap Materials, Ministry of Education, School of Physics and Electronic Engineering, Harbin Normal University, Harbin, 150025, Heilongjiang Province, China. E-mail: yaochengbao5@163.com 


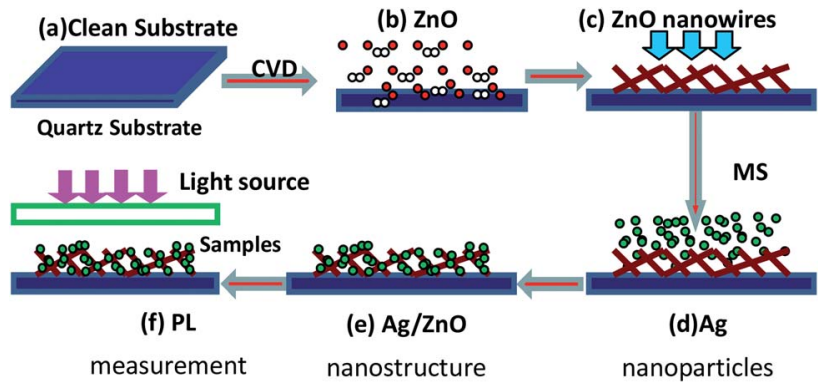

Scheme 1 Fabrication of the $\mathrm{Ag}-\mathrm{ZnO}$ nanowires: (a) quartz substrate cleaned using the modified dip-coating method; (b) $\mathrm{ZnO}$ nanowires etched with Ar carrier gas; (c) as-deposited ZnO nanowires; (d) Ag nanoparticles produced for different sputtering times; (e) the Ag/ZnO nanostructure; (f) PL measurement.

have been successfully synthesized; their morphology, structure and nonlinear optical properties are investigated. The results show that the samples have excellent structures and properties and thus, they are expected to have potential applications in photoelectric devices.

\section{Experiment}

The $\mathrm{Ag}-\mathrm{ZnO}$ nanowires were grown on quartz substrates by twostep chemical vapour deposition (CVD) and magnetron sputtering (MS) method. The schematic of the process is depicted in Scheme 1 . The quartz substrate was cleaned ultrasonically in acetone, rinsed in deionized water and subsequently dried in flowing nitrogen gas before deposition. Then, the $\mathrm{ZnO}$ nanowires were grown by CVD, and Ag nanoparticles were deposited on the quartz substrates with ZnO nanowires by using the MS system.

Fig. 1 shows the experimental setup for our CVD and MS systems. In Fig. 1(a), IG, OG and QT denote the input gas, output gas, quartz tube, respectively. SP and S are the source powder and substrates, respectively. In Fig. 1(b), RFT is a radio frequency target with a disc of $\mathrm{Ag} 60 \mathrm{~mm}$ in diameter and purity of $99.99 \%$. S stands for substrates. $\mathrm{ZnO}(1 \mathrm{~g})$ powder was used as the precursor, and it was placed in an alumina boat and loaded into the tubular furnace. The Au-coated quartz substrates were placed downstream from the powders. The furnace was heated to a preset temperature of $1250^{\circ} \mathrm{C}$. Ar was used as the carrier gas during growth at a constant flow rate of $100 \mathrm{sccm}$ and pressure of $50 \mathrm{~Pa}$. Then, the furnace was cooled down naturally to room temperature. During deposition, the base pressure in the deposition chamber and the radio frequency power were $6.0 \times$ $10^{-4} \mathrm{~Pa}$ and $100 \mathrm{~W}$, respectively. The sample was grown in an ambience with Ar sputtering at $20 \mathrm{sccm}$ and a constant working pressure of 1.0 Pa.

The structural properties of $\mathrm{Ag}-\mathrm{ZnO}$ and $\mathrm{ZnO}$ nanowires were observed by field emission scanning electron microscopy (FE-SEM). Crystallinity was analyzed using an X-ray diffractometer (XRD) equipped with monochromated $\mathrm{Cu} \mathrm{K \alpha}$ irradiation. RTPL and LTPL spectra were obtained to study the optical properties of the samples; the excitation laser was a $\mathrm{He}-\mathrm{Cd}$ laser with a wavelength of $325 \mathrm{~nm}$, and the spectra were obtained from $350 \mathrm{~nm}$ to $800 \mathrm{~nm}$. The nonlinear optical properties of $\mathrm{Ag}-\mathrm{ZnO}$ nanowires were investigated by performing the femto-, pico- and nano-second $Z$-scans, as described previously. ${ }^{29-32}$ The samples were pumped at $532 \mathrm{~nm}$ by frequencydoubled 100 fs pulses from an amplified Ti-sapphire laser (1 $\mathrm{kHz}$ ) and the second harmonic wavelengths of a pico- and nanosecond Nd: YAG laser (Continuum Model YG 601, $50 \mathrm{~Hz}$ and 10 $\mathrm{Hz}$ ), which provided pulses of about $21 \mathrm{ps}$ and $4 \mathrm{~ns}$. The output pulse energies from the laser system were $0.4 \mathrm{~mJ}, 0.6 \mathrm{~mJ}$ and 1.0 $\mathrm{mJ}$ for $4 \mathrm{~ns}, 3.0 \mathrm{~J}, 8.4 \mathrm{~J}$ and $16.8 \mathrm{~J}$ for $21 \mathrm{ps}$ and $42.6 \mathrm{~J}, 58.4 \mathrm{~J}$ and $74.6 \mathrm{~J}$ for $100 \mathrm{fs}$ with linear polarization. Time-resolved transient absorption spectra were recorded using a femtosecond two color pump-probe (TCPP) spectrometer, which has been described in detail elsewhere. ${ }^{33,34}$ Briefly, a Ti: sapphire laser system, same as the above-mentioned femtosecond $Z$-scan system, was used. The pump-induced absorption changes were probed over the range from 450 to $750 \mathrm{~nm}$ with a femtosecond white-light continuum generated in the sapphire plate. All experiments were performed at room temperature.

\section{Results and discussion}

\subsection{Morphologies and structures of $\mathrm{Ag}-\mathrm{ZnO}$ nanowires}

Fig. 2 (M1, M = a-d) shows the top-view FE-SEM images of the unmodified $\mathrm{ZnO}$ and $\mathrm{Ag}-\mathrm{ZnO}$ nanowires grown on the quartz substrates. The inset of Fig. 2 (M1) shows the size distribution diagram of $\mathrm{ZnO}$ nanowires and $\mathrm{Ag}$ nanoparticles for each sample. The $\mathrm{Ag}-\mathrm{ZnO}$ nanowires exhibited rougher surfaces because of the adsorption of $\mathrm{Ag}$ nanoparticles with the diameter of $\sim 34.86 \mathrm{~nm}$ (scale bar: $50 \mathrm{~nm}$ ); Fig. 2 (M2) shows magnified SEM images of such structures. As the evaporation time was
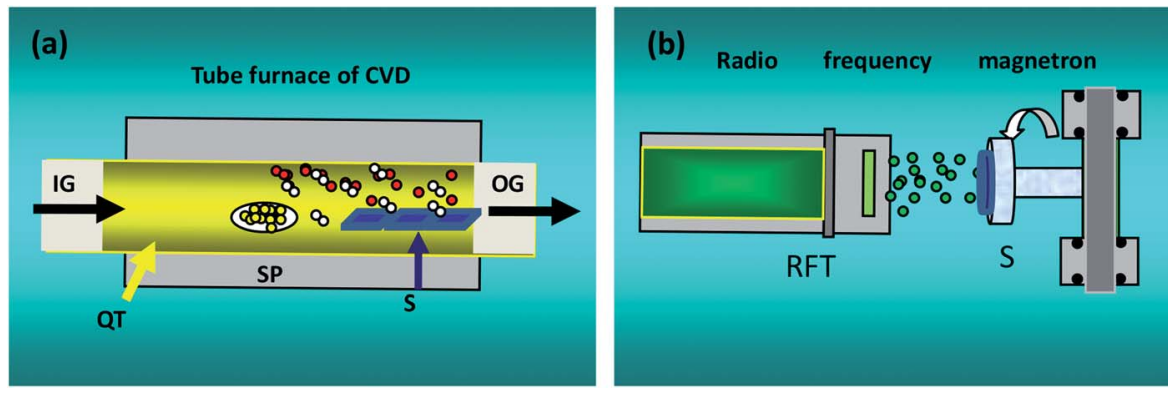

Fig. 1 Conceptual illustration for CVD and MS experiments. 

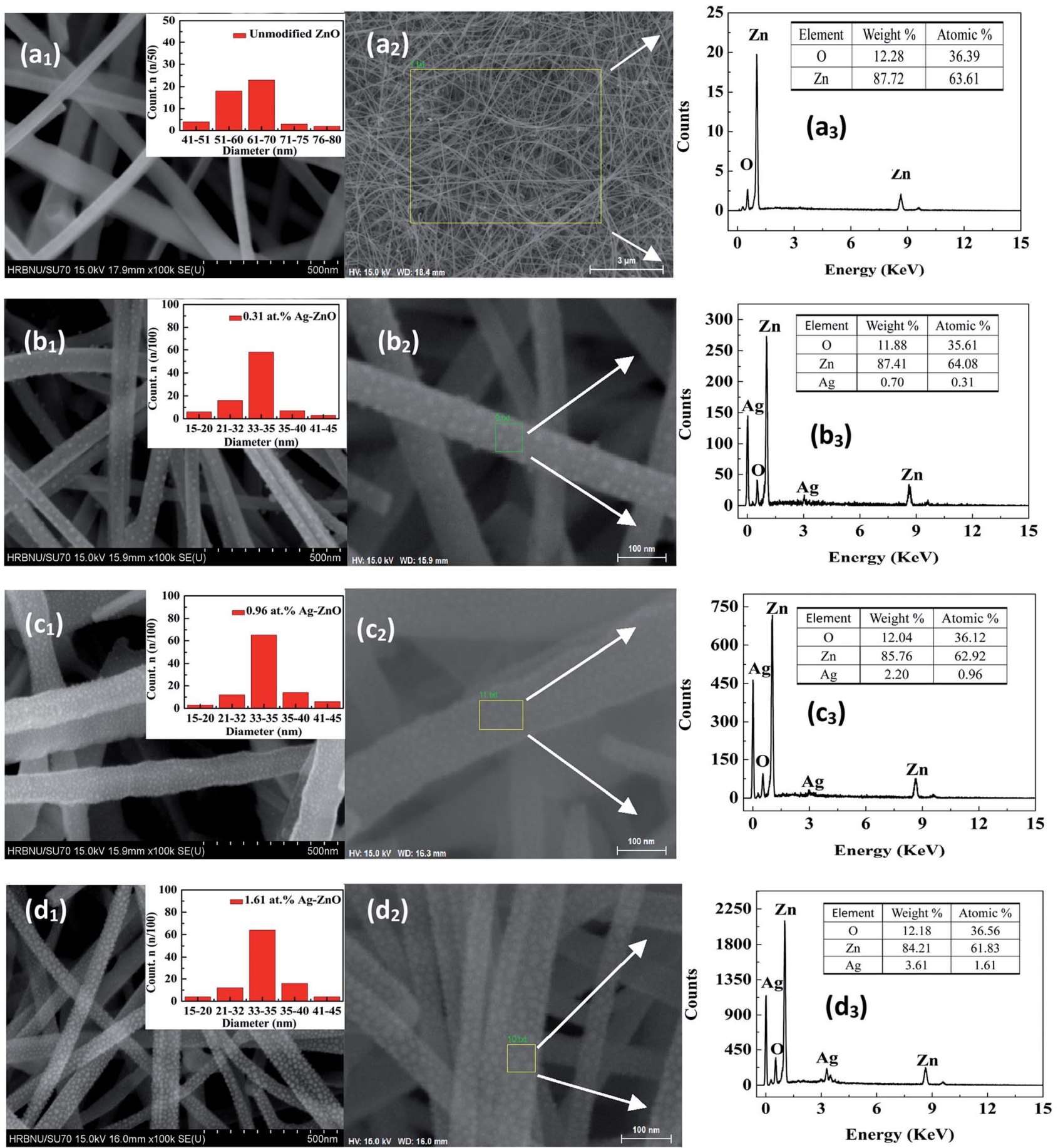

Fig. 2 Top-view SEM images (a1-d1), magnified SEM images (a2-d2), EDX spectra (a3-d3) for Ag-ZnO nanowires with sputtering times of 0 s, $80 \mathrm{~s}, 100 \mathrm{~s}$, and $120 \mathrm{~s}$, respectively.

increased, the atomic percent of $\mathrm{Ag}$ gradually increased. $\mathrm{A}$ typical energy dispersive X-ray (EDX) spectrum of the Ag-ZnO nanowires is plotted in Fig. 2 (M3). Clear peaks of oxygen, Zn, and $\mathrm{Ag}$ were observed in EDX spectra. The EDX spectroscopy data confirmed that all samples contained $0.31 \%, 0.96 \%$ and $1.61 \%$ silver.

XRD analysis was carried out to investigate the structure of $\mathrm{Ag}-\mathrm{ZnO}$ nanowires. As shown in Fig. 3(a-d), all patterns revealed a strong characteristic peak for the (002) plane of the ZnO hexagonal wurtzite structure. The other diffraction peaks were caused by the presence of $\mathrm{Ag}$ and $\mathrm{Zn}$ in the synthesized films. The peaks at two theta values of 38.24 degree could be assigned to the (111) plane of the face-centered-cubic structure of Ag. Therefore, these characteristic peaks appeared in the Xray diffraction pattern due to the formation of a binary phase consisting of wurtzite $\mathrm{ZnO}$ and face-centered-cubic Ag. 

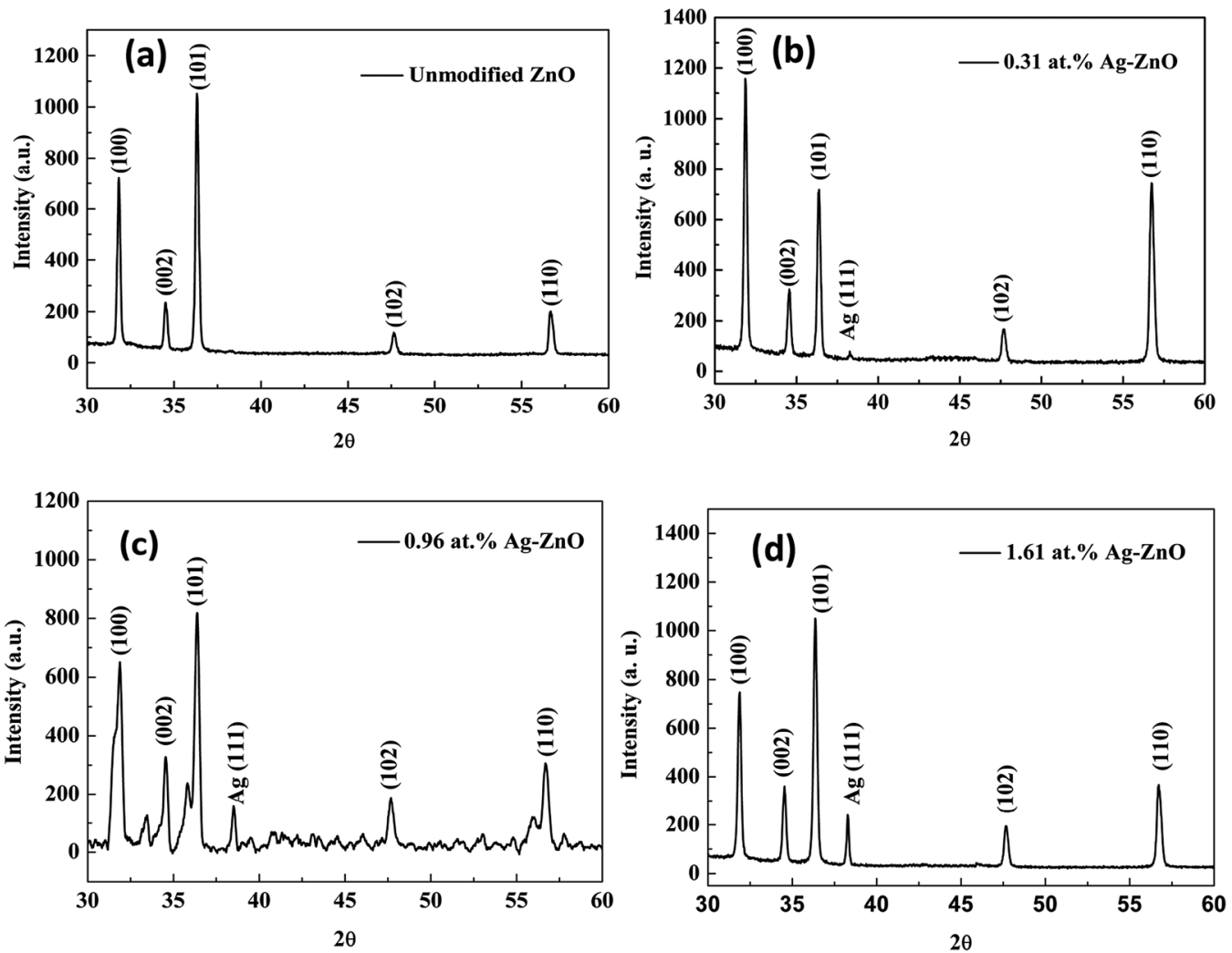

Fig. 3 XRD patterns (a-d) for Ag-ZnO nanowires with sputtering times of 0 s, $80 \mathrm{~s}, 100 \mathrm{~s}$, and $120 \mathrm{~s}$, respectively.

The TEM images of the Ag-ZnO nanowires synthesized at sputtering times of $80 \mathrm{~s}, 100 \mathrm{~s}$, and $120 \mathrm{~s}$ are presented in Fig. 4(a-c), respectively. Fig. 4(d) shows that the average diameters of $\mathrm{Ag}$ nanoparticles for different times are similar, but the densities are different. Fig. 4(e) and (f) show the HRTEM and selected-area electron diffraction (SAED) patterns of the Ag-ZnO nanowires synthesized at $100 \mathrm{~s}$, respectively. These results indicated that the $\mathrm{ZnO}$ nanowires with $0.32 \mathrm{~nm}$ space between the adjacent lattice planes had high crystal quality and were highly oriented to the $c$-axis direction of the 0002 crystal. (a)

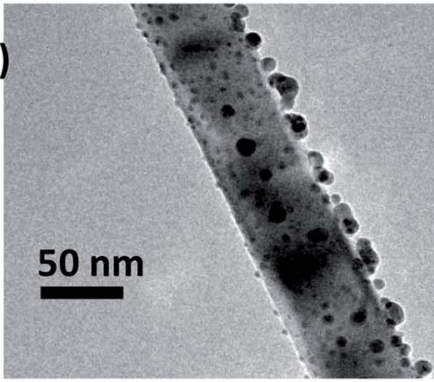

(b)

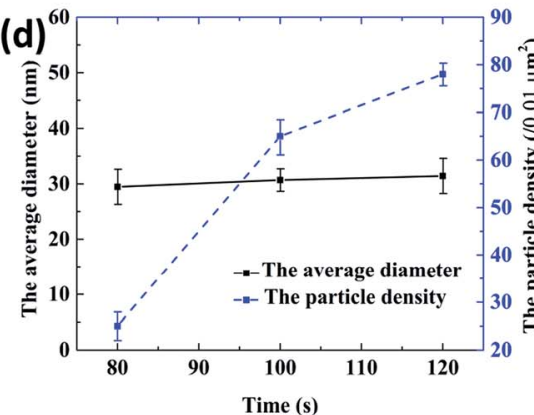

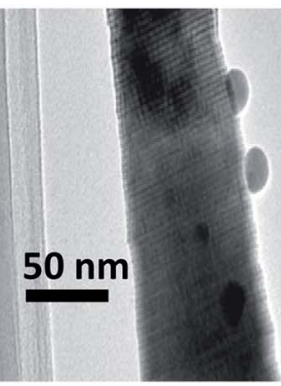

(e)

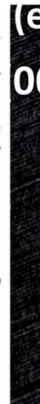

(c)

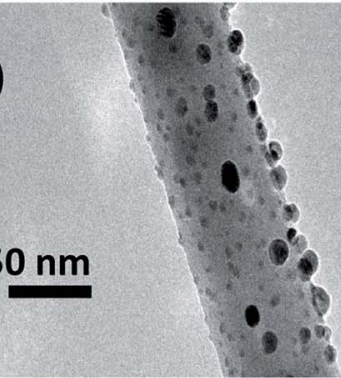

(f)

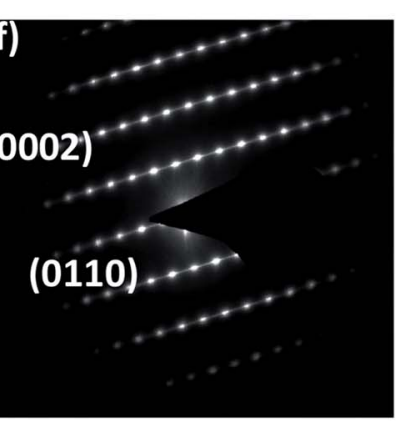

Fig. 4 TEM of Ag-ZnO nanowires with sputtering times of (a) $80 \mathrm{~s}$, (b) $100 \mathrm{~s}$, and (c) $120 \mathrm{~s}$; (d) Average diameter and particle density of Ag-ZnO nanowires with sputtering times of $80 \mathrm{~s}, 100 \mathrm{~s}$, and $120 \mathrm{~s}$. HRTEM (e) and SAED image (f) of the Ag-ZnO nanowires for the sputtering time of $100 \mathrm{~s}$. 


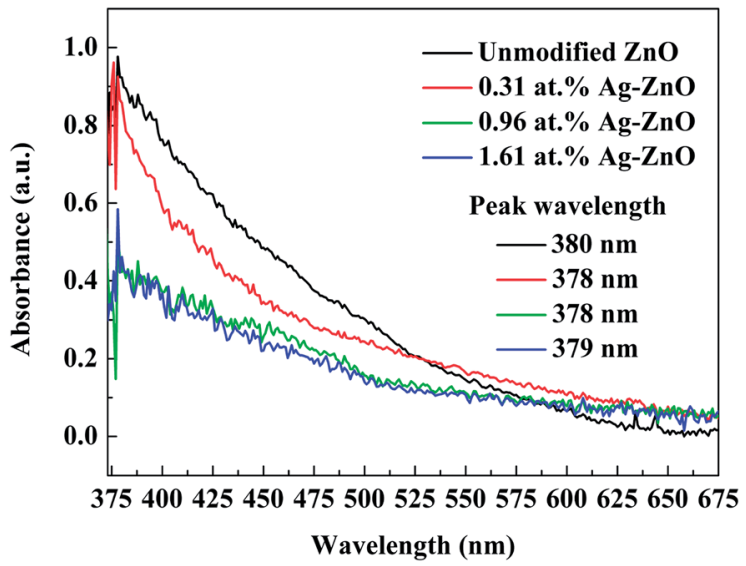

Fig. 5 UV-visible spectra of $\mathrm{Ag}-\mathrm{ZnO}$ nanowires with sputtering times of $0 \mathrm{~s}, 80 \mathrm{~s}, 100 \mathrm{~s}$, and $120 \mathrm{~s}$.

\subsection{Steady-state absorption spectra}

The UV-visible spectra of $\mathrm{Ag}-\mathrm{ZnO}$ nanowires prepared with different sputtering times are shown in Fig. 5. Clearly, all the samples exhibited strong linear absorption bands with a peak in the near-UV range (peak wavelength at 380, 378, 378 and $379 \mathrm{~nm}$ ). The Ag-ZnO nanowires showed sharp absorptions at wavelengths slightly lower than $380 \mathrm{~nm}$, and the addition of silver did not result in any significant difference in the band gap. Furthermore, silver-modified $\mathrm{ZnO}$ seed layer films with different contents of Ag were employed to corroborate the SPR absorption of Ag nanoparticle. The decoration of Ag nanoparticle induced band tail state in the Ag-ZnO nanowires. ${ }^{35}$ In comparison with the spectra of unmodified $\mathrm{ZnO}$ nanowires, the spectra of $\mathrm{Ag}-\mathrm{ZnO}$ nanowires exhibited narrower peaks, and we found that the absorption rate of the $\mathrm{Ag}-\mathrm{ZnO}$ nanowires was reduced.

\subsection{RTPL and LTPL spectra of Ag-ZnO nanowires}

To study the PL characteristics, we recorded RTPL and LTPL spectra of unmodified $\mathrm{ZnO}$ and $\mathrm{Ag}-\mathrm{ZnO}$ nanowires at different temperatures. Fig. 6(a) shows that all samples emitted strongly at $380.6,378.6,378.5$ and $379.8 \mathrm{~nm}$ with sharp ultraviolet (UV) emission peaks (see Fig. 6(c)), corresponding to the near band edge (NBE) emission. Another broad peak in the visible range of 450-650 nm originated from the electron-hole recombination at a deep level (DL), which was caused by oxygen vacancy or zinc interstitial defects. ${ }^{36,37}$ After decoration with the $\mathrm{Ag}$ nanoparticles, the intensity of the visible emission peaks increased. According to some reports, this longer wavelength emission is due to the excitation caused by the recombination of photogenerated holes with singly ionized $\mathrm{O}_{\mathrm{v}}$ site ${ }^{37}$ The ultraviolet emission peaks are derived from the radiated composite luminescence of the forbidden band free excitons, and the peak of blue light emission is related to DL emission (for example, the electron transport from $\mathrm{Zn}_{\mathrm{i}}$ leads to $\mathrm{V}_{\mathrm{Zn}}$ electron transition luminescence), as reported in many papers. ${ }^{37,38}$ In Fig. 6(a), it can be clearly seen that the peak intensity ratio of DL emission to NBE emission follows the order $\mathrm{Ag}-\mathrm{ZnO}$ nanowires > unmodified $\mathrm{ZnO}$ nanowires, which can be explained by a combination of a DL emission enhancing effect. The electron density of the Ag nanoparticles increased due to the transfer of electrons from the conduction band ( $\mathrm{CB}$ ) of $\mathrm{ZnO}$ nanowires to the Fermi level (FL) of Ag nanoparticles. ${ }^{38}$ In addition, it should also be pointed out that the DL emission peak of the unmodified $\mathrm{ZnO}$ nanowires was very weak compared to that of the Ag$\mathrm{ZnO}$ nanowires. The electrons in the FL of $\mathrm{Ag}$ nanoparticles were excited due to surface plasmon resonance (SPR) of $\mathrm{Ag}$ nanoparticles upon interaction with the incident photon. These excited electrons may be transferred to the defect levels of $\mathrm{ZnO}$. Since the FL of $\mathrm{Ag}$ nanoparticles is higher than the defect level of $\mathrm{ZnO}$, the defect level electrons could recombine with the hole in the valence band of the $\mathrm{ZnO}$ nanowires. The $\mathrm{ZnO}$ PL bandedge enhancement due to both charge-transfer mechanisms and local-field mechanisms created a fundamental problem in studying the exciton-plasmon interactions using rough metal films as the source of plasmonic interactions. In Fig. 6(b) and
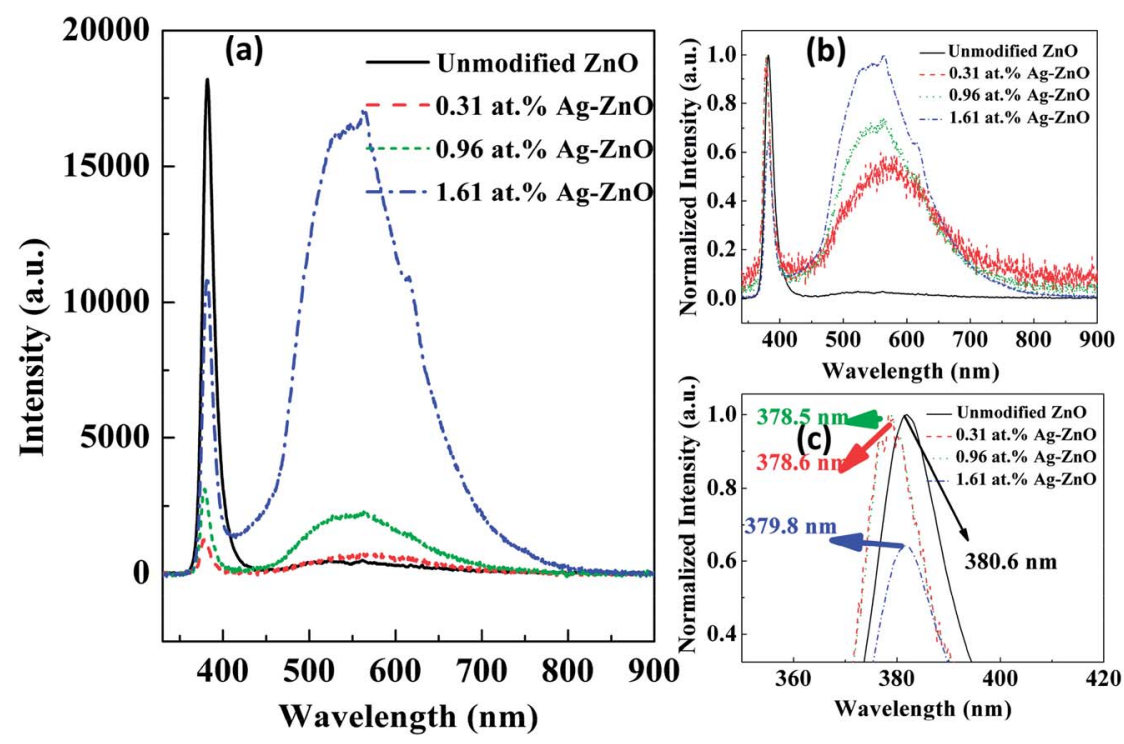

Fig. $6 \mathrm{PL}(\mathrm{a})$ and normalized PL spectra (b and c) of unmodified $\mathrm{ZnO}$ and $\mathrm{Ag}-\mathrm{ZnO}$ nanowires. 
(c), we can observe that the positions of the UV and visible emission peaks are slightly changed. The smaller blue shift in the UV emission resulted from the band-side excitation of the semiconductor, which generally occurs near the surface of the sample, resulting in uneven distribution of the excited carriers. To maintain a balanced and uniform distribution, excess unbalanced carriers diffused from the surface to the interior and simultaneously recombined due to radiative or nonradiative transition processes. Therefore, the majority of the excitations in the crystal were confined to the penetration depth of the photons below the surface. The red shift in the visible emission resulted from the intrinsic defects or/and Ag nanoparticles. Similar shifts in $\mathrm{Ag} / \mathrm{ZnO}$ have been reported by $\mathrm{L} . \mathrm{Su}$ et $a .^{35}$ and M. Liu et $a .^{40}$

Fig. 7(a) shows the LTPL spectra of the Ag-ZnO nanowires in the range of 30-250 K. Each curve was fitted as a weighted sum of three components with centers at $3.374,3.364$, and $3.326 \mathrm{eV}$. The $3.376 \mathrm{eV}$ peak is caused by free exciton $(\mathrm{FX}=\mathrm{NBE})$ recombination. The peaks at 3.364 and $3.326 \mathrm{eV}$ can be due to bound excitons (DE). Similar results have been obtained by A. Amutha et $a l^{38}$ for Ag-decorated $\mathrm{ZnO}$ nanorods, and a similar phenomenon has been obtained by H. P. He et al. ${ }^{39}$ for Al-doped ZnO. In addition, the LTPL spectra exhibit an NBE emission in the UV region (Fig. 7(b)) and DL emission in the visible region (Fig. 7(c)). At room temperature, the NBE emission peaks are observed at $3.264 \mathrm{eV}(379 \mathrm{~nm})$. In Fig. 7(b), we can see that with the increasing temperature, the intensity of the emission peak in the UV region decreases drastically. When the temperature exceeds $120 \mathrm{~K}$, the dominant emission becomes an A line. As the temperature further increases, the first LO-phonon replica gradually merges into the A line, resulting in marked broadening of the NBE emission. The relative intensity of the DL emission peaks at about $2.20 \mathrm{eV}$ is strongly reduced; the wavelength locations do not shift. No clear change is observed in the peak position of visible emission in the RTPL spectra.

The ZnO band and the NBE and DL emissions are illustrated in Fig. 8. The process of electron dynamics can be explained as follows: the electrons get excited from the valence bands of $\mathrm{ZnO}$ nanowires to the conduction band by absorbing the incident laser photons, the near band edge (NBE) emission, and the electron transfer from the conduction band of $\mathrm{ZnO}$ nanowires to the Fermi level of Ag nanoparticles. These electrons are excited due to interactions between surface plasmon resonance (SPR) of $\mathrm{Ag}$ nanoparticles and the incident photons. These excited electrons may be transferred to the defect levels of $\mathrm{ZnO}$. Since the Fermi level of $\mathrm{Ag}$ nanoparticles is higher than the defect level of $\mathrm{ZnO}$, the defect level electrons can recombine with the hole in the valence band of $\mathrm{ZnO}$ nanowires. The visible emission peak is observed in the $\mathrm{Ag}-\mathrm{ZnO}$ nanowire RTPL spectra. As shown in Fig. 8 (CII and CIII), all emission peaks can be analyzed by considering the energy band diagram. According to some reports, this long wavelength emission is excited by the recombination of photogenerated holes with the singly ionized oxygen vacancy sites. ${ }^{41-46}$ The UV emission peak is derived from
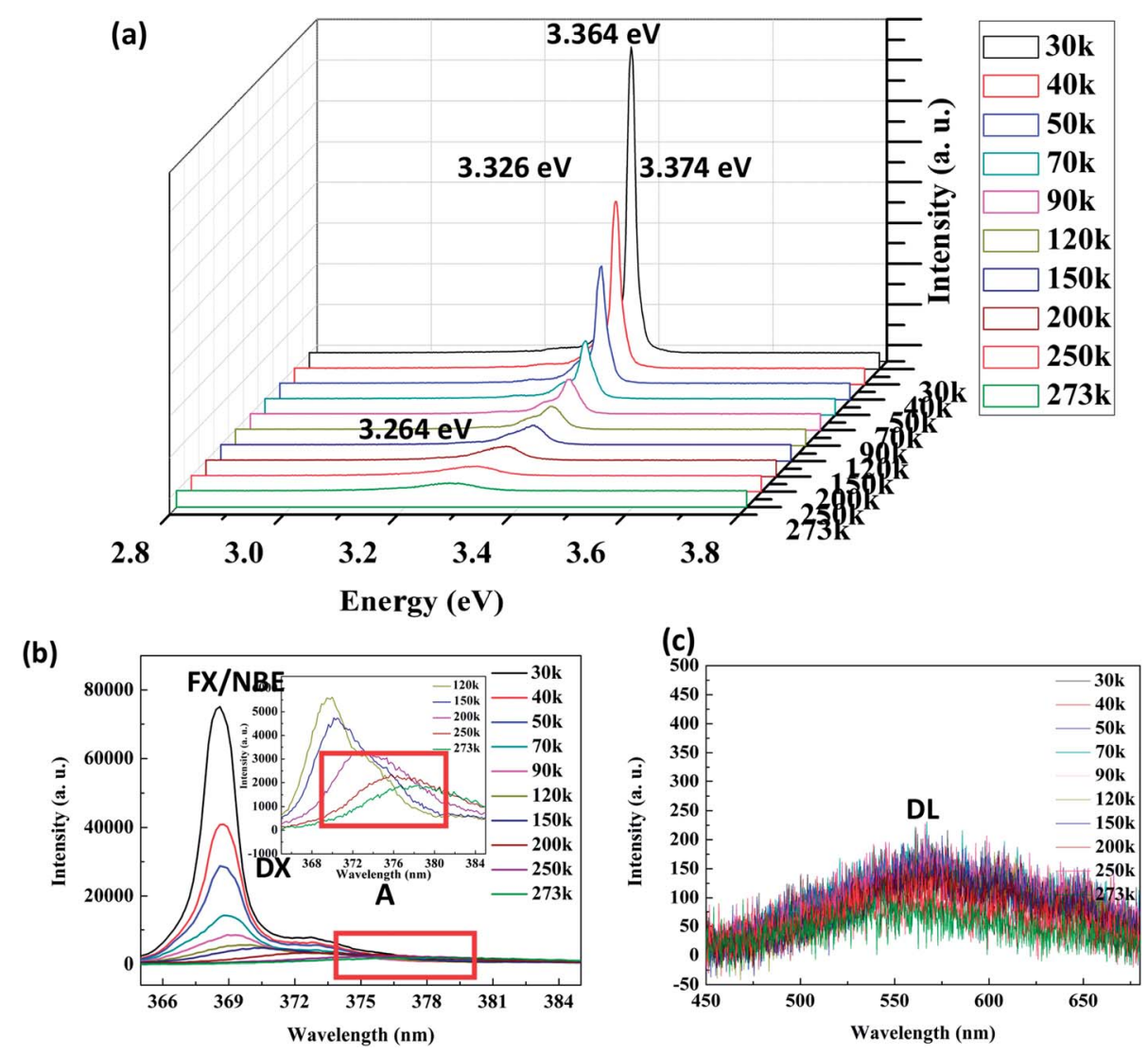

Fig. 7 LTPL spectra (a), ultraviolet (b) and visible (c) region spectra of Ag-ZnO nanowires with sputtering time of $120 \mathrm{~s}$. 


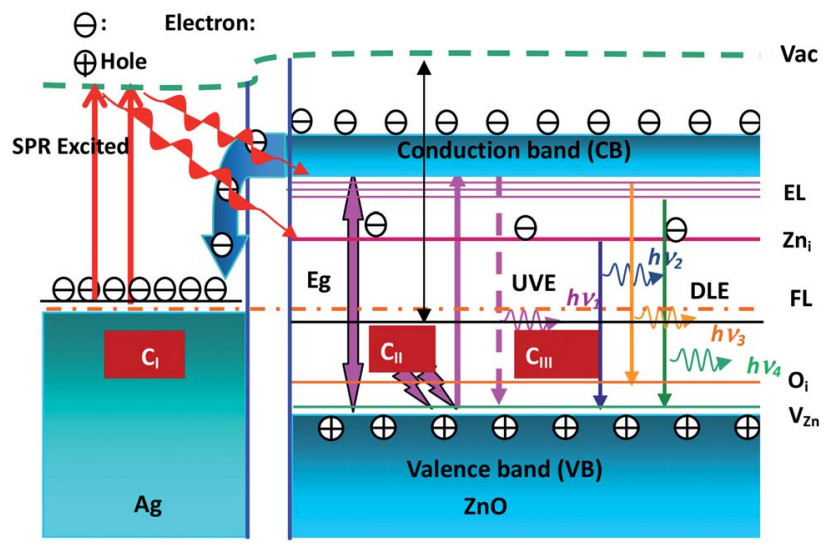

Fig. 8 Illustration of the $\mathrm{ZnO}$ band $\left(E_{\mathrm{g}}\right)$, energy level ( $\mathrm{CB}$ and $\left.\mathrm{VB}\right)$ of $\mathrm{ZnO}$, excitation levels (EL) defects $\left(\mathrm{Zn}_{\mathrm{i}}, \mathrm{O}_{\mathrm{i}}\right.$ and $\left.\mathrm{V}_{\mathrm{Zn}}\right)$, fermi levels $(\mathrm{FL})$ of Ag, ultraviolet (UV) and deep-level (DL) emission.

the radiated composite luminescence of the forbidden band free excitons, and the peak of blue light emission originates from the electron transition from $\mathrm{Zn}_{\mathrm{i}}$ to zinc vacancy $\left(\mathrm{V}_{\mathrm{Zn}}\right)$ or from the $\mathrm{Zn}_{\mathrm{i}}$ level to the top of the VB. As shown in Fig. 8 (CI), the electron density of $\mathrm{Ag}$ nanoparticles increases due to the transfer of electrons from the $\mathrm{CB}$ of $\mathrm{ZnO}$ nanowires to the Fermi level (FL) of Ag nanoparticles. These electrons are excited by the interactions between the SPR effects of $\mathrm{Ag}$ nanoparticles (around $\mathrm{UV}^{47}$ ) and the incident photons. These excited electrons may be transferred to the DL of ZnO nanowires, and they may recombine with the holes in the $\mathrm{VB}$ of $\mathrm{ZnO}$ nanowires since the $\mathrm{FL}$ of Ag nanoparticles is higher than the DL of ZnO. Therefore, the DL emission is enhanced in $\mathrm{Ag}-\mathrm{ZnO}$ nanowires. Thus, as shown in Fig. 6(a), with the decorated Ag nanoparticles, the intensities of the visible emission peaks increase.

For LTPL spectra, when the laser light (325 $\mathrm{nm}$ excitation) is incident on the $\mathrm{Ag}-\mathrm{ZnO}$ nanowires, the electrons in the VB are excited to the $\mathrm{CB}$. Then, the electrons in the $\mathrm{CB}$ of $\mathrm{ZnO}$ nanowires are transferred to the FL of Ag nanoparticles. Hence, the electron density increases in the FL of Ag nanoparticles. These electrons are excited and transferred to the bottom of the $\mathrm{CB}$ and recombine with the holes in the $\mathrm{VB}$ of $\mathrm{ZnO}$ nanowires, producing radiative emission. Therefore, the band edge emission increases when $\mathrm{Ag}$ nanoparticles are in contact with $\mathrm{ZnO}$ nanowires. The enhancement in PL emission is the combined effect of SPR of Ag nanoparticles coupled with both the excitons and defects in the $\mathrm{ZnO}$ nanowires.

\subsection{The nonlinear optical properties of $\mathrm{Ag}-\mathrm{ZnO}$ nanowires}

Fig. 9(a1-c1) and (a2-c2) show the curves of the open- and closeaperture $Z$-scans, respectively. It can be seen from the transmittance curve of the closed-aperture $Z$-scan that the signal profile has a valley followed by a peak, indicating positive (selffocusing) optical nonlinearity. The nonlinear absorption behavior of $\mathrm{Ag}-\mathrm{ZnO}$ nanowires is revealed using a $532 \mathrm{~nm}$ laser irradiation; upon excitation, the CB was populated through the process of two-photon absorption (TPA). Because the laser excitation wavelength falls in the wing region of the exciton absorption, the condition for nonlinear absorption by TPA process $\left(E_{\mathrm{g}} / 2<\hbar \omega<E_{\mathrm{g}}\right.$, in Fig. 4) was satisfied by the TPA process and the strong absorption of the defect states. Under different laser energies, the $\mathrm{Ag}-\mathrm{ZnO}$ nanowires exhibited different nonlinear absorption mechanisms. The low-energy
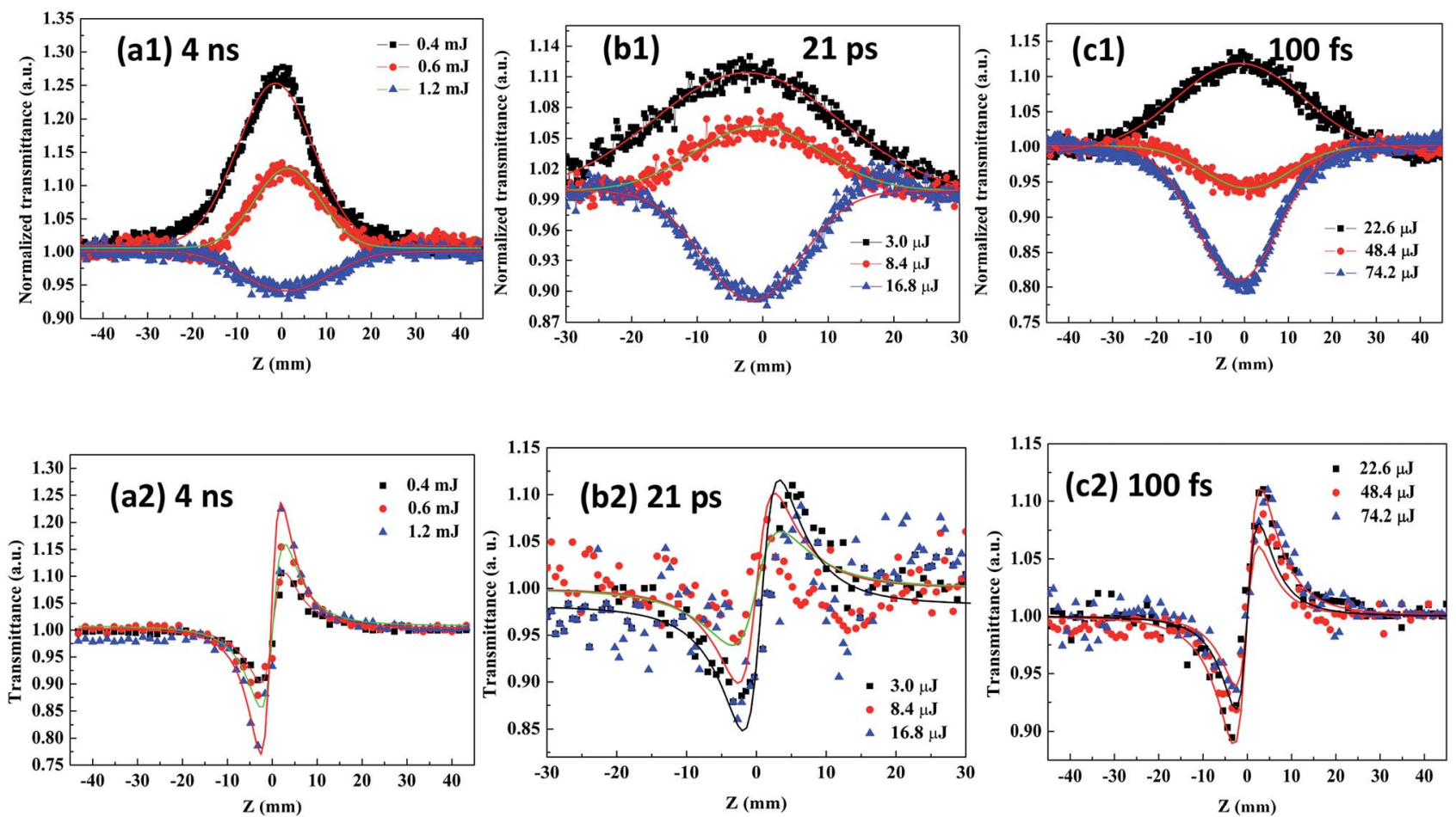

Fig. 9 Normalized open- (a1-c1) and closed- (a2-c2) aperture Z-scan transmittance curves of Ag-ZnO nanowires measured at $532 \mathrm{~nm}$ with 4 ns, 21 ps and 100 fs pulse width, respectively. 
$\mathrm{Ag}-\mathrm{ZnO}$ nanowires displayed a normalized transmittance peak at the beam focus, indicating negative nonlinear absorption due to typical two - photon excitation-induced ground state saturable absorption (SA). However, the high-energy Ag-ZnO nanowires demonstrated a strong positive nonlinear absorption (reverse saturable absorption, RSA) in addition to SA, and the nonlinear absorption completely switched over to RSA. The change from SA to RSA near the focal region of the beam can be interpreted by considering the intensity-dependent contributions from SA and RSA. With an increase in the excitation power, the intensity of SA in the ground state reduced. When the excitation power was relatively large, a "valley" curve (near the focal point of the transmittance decreases), which is typical of the two - photoninduced excited state RSA effect, was obtained. The theoretical explanation of the mechanism of transformation is as follows: since the maximum power density of the probe beam occurred at the focal point of the lens, the nonlinear absorption of the sample was the strongest. For the two photon excitation-induced ground state SA, the electrons in the ground state were excited to the excited state; when there are no more electrons to absorb the extra photons, the phenomenon is called the "ground bleaching effect". Therefore, the closer the focal point, the higher the transmittance, and it will appear as the "peak" in the measurement curve. In TPA-induced RSA, the sample first absorbed two photons at a time, which led to lower transmittance; the more closer it gets to the focal point, the more clear the effect, represented by the "valley" of the curve.

In the case of a thin saturable absorber, the optical intensity loss, as the beam propagates through the sample, is given by the relation ${ }^{48-50}$

$$
\frac{\mathrm{d} I}{\mathrm{~d} z}=-\alpha(I) I
$$

where $z$ is the propagation distance, $I$ is the incident intensity in the sample, and the total absorption coefficient $\alpha(I)$ is now given as $^{51,52}$

$$
\alpha(I)=\frac{\alpha_{0}}{1+\left(I / I_{\mathrm{S}}\right)}+\beta I
$$

where $\alpha_{0}\left(\alpha_{0}=4.37 \mathrm{~cm}^{-1}\right)$ is the linear absorption coefficient at the wavelength of excitation, $I_{\mathrm{S}}=\left(h \nu / \sigma_{0} \tau\right)$ is the saturation intensity, $h \nu$ is the photon energy, and $\tau$ is the lifetime of the excited state. Saturation intensity is the intensity at which the absorption coefficient drops to half of its linear value. $\beta$ is the third order nonlinear absorption coefficient. The first term of eqn (2) describes negative nonlinear absorption (SA); the second term describes positive nonlinear absorption (RSA) mediated by the excited state (include defect states), with relatively smaller contribution from TPA. The transmission of the saturable absorber is given by the equation ${ }^{53-55}$

$$
T=\exp \left(-\alpha_{0} L_{\mathrm{eff}}\left(1-\frac{I}{I_{\mathrm{S}}}\right)\right)
$$

The values of the nonlinear absorption and refractive index coefficients were expressed using the relation ${ }^{56-59}$

$$
\begin{gathered}
T(z, S=1)=\sum_{m}^{\infty}\left(\left[-q_{0}\right]^{m} /(m+1)^{3 / 2}\right) \\
\Delta T_{\mathrm{p}-\mathrm{v}}=0.406(1-S)^{0.25}\left|\phi_{0}\right|
\end{gathered}
$$

where $q_{0}(z)=\beta I_{0} L_{\mathrm{eff}} /\left(1+z / z_{0}\right), z_{0}$ is the Rayleigh range, $L_{\mathrm{eff}}=(1-$ $\left.\exp \left(-\alpha_{0} L\right)\right) / \alpha_{0}$ is the effective length with $L$ being the thickness of the sample. $\Delta T_{\mathrm{p}-\mathrm{v}}$ is the measured peak-valley transmittance difference, $\Delta \phi_{0}=k n_{2} I_{0} L_{\text {eff }}$ is the on-axis phase-shift and $I_{0}$ is the incident intensity at focus. $S$ is the linear transmittance of the aperture given by $S=1-\exp -2\left(r_{\mathrm{a}} / w_{\mathrm{a}}\right)^{2}$, where $r_{\mathrm{a}}$ is the radius of the aperture and $w_{\mathrm{a}}$ is the radius of the laser spot before the aperture. The values of $\beta$ and $n_{2}$ were evaluated by fitting the experimental data to eqn (4) and (5). The nonlinear absorption coefficients and nonlinear refractive index are shown in Table 1. Due to the presence of $\mathrm{Ag}$, the particles that were excited rapidly relaxed to the FL of $\mathrm{Ag}$. In addition, the FL of $\mathrm{Ag}$ is higher than the FL of $\mathrm{ZnO}$, making the electrons relax nonradiatively to the FL of $\mathrm{ZnO}$ and promoting RSA of the excited state. Similar analysis has been reported in many papers. ${ }^{59-62}$ For example, $\mathbf{M}$. T. Cisneros et $a .^{59}$ and R. Sato et $a l .{ }^{60}$ reported the nonlinear optical behavior of Ag nanoparticles in PMMA; Y. P. Chan et al. ${ }^{61}$ and T. Voss et al. ${ }^{62}$ reported the nonlinear optical properties of $\mathrm{ZnO}$. Compared with the Ag nanoparticles or pure $\mathrm{ZnO}$ nanowires, the $\mathrm{Ag}-\mathrm{ZnO}$ nanowires exhibited stronger nonlinear absorption. In contrast, the nonlinear absorption coefficient of the IZO nanowires was larger than the previously reported values of $\mathrm{Ag}$ nanoparticles and $\mathrm{ZnO}$.

To discuss various parameters in the ns, ps and fs regimes that influence the RSA and SA, a general model of the multi-level energy diagram ${ }^{\mathbf{6 3 , 6 4}}$ is used. For the nano- and pico-second laser pulses, three level models were adopted for analysis. Therefore, the nonlinear absorption mechanism of the $\mathrm{Ag}-\mathrm{ZnO}$ nanowires can be explained by the repopulation of $\mathrm{CB}$ from the VB caused by TPA-induced ground state SA and TPA-induced low excited state (or DL) RSA. For the femtosecond laser, four level model analyses were carried out. The nonlinear absorption mechanism of the $\mathrm{Ag}-\mathrm{ZnO}$ nanowires should be repopulation of $\mathrm{CB}$ from the VB caused by TPA-induced low excited state (or DL) SA and TPA-induced high excited state (CB) RSA. The abovementioned process is shown in Fig. 10. The electrons get to the excited state from the ground state by absorbing the

Table 1 Third-order nonlinear optical coefficients of Ag-ZnO nanowires at the laser pulse of $5 \mathrm{~ns}, 21 \mathrm{ps}$, and $100 \mathrm{fs}$, respectively

\begin{tabular}{llr}
\hline Laser pulse & $\beta$ & $n_{2}$ \\
\hline $4 \mathrm{~ns}$ & $-24.2,-42.5,78.8\left(\mathrm{~cm} \mathrm{GW}^{-1}\right)$ & $4.4,5.2,7.8\left(\times 10^{-17} \mathrm{~m}^{2} \mathrm{~W}^{-1}\right)$ \\
$21 \mathrm{ps}$ & $-32.8,-54.6,104.3\left(\mathrm{~cm} \mathrm{GW}^{-1}\right)$ & $4.7,5.9,8.9\left(\times 10^{-17} \mathrm{~m}^{2} \mathrm{~W}^{-1}\right)$ \\
$100 \mathrm{fs}$ & $-38.2,68.5,138.5\left(\mathrm{~cm} \mathrm{GW}^{-1}\right)$ & $5.2,7.4,11.3\left(\times 10^{-17} \mathrm{~m}^{2} \mathrm{~W}^{-1}\right)$
\end{tabular}




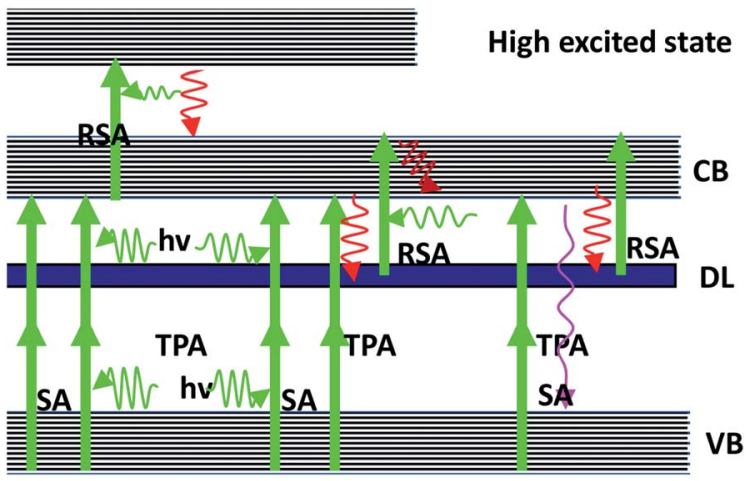

(I)

(III)
Fig. 10 Multi-level energy diagram showing VB and $D L, C B$ and singlet high excited states, respectively. The solid lines and dashed lines represent optical excitation and nonradiative relaxation, respectively.

incident laser photons. These excited electrons relax to the $\mathrm{S}_{1}$ state in fs time scale by nonradiative decay. The excited electrons may also exhibit transitions to DL by the mechanism of nonradiative relaxation within the ps time scale. The electrons in DL can be further excited to the CB state. This process is known as the excited state absorption (ESA). On ps time scales, singlet-singlet transitions result in the ESA process.

\subsection{Transient absorption spectra of $\mathrm{Ag}-\mathrm{ZnO}$ nanowires}

Fig. 11 shows the TCPP experimental results. The samples were excited at different wavelengths $(325,380$ and $400 \mathrm{~nm})$ and probed at different wavelengths $(480.18,500.41,550.39,600.37$, and $640.83 \mathrm{~nm}$ ). The right panel shows the results at $325 \mathrm{~nm}$; the middle and left panel show the results at 380 and $400 \mathrm{~nm}$, respectively. The results in Fig. 11 are presented in the full time window up to $1.2 \mathrm{~ns}$. This sample, was after being excited with $325 \mathrm{~nm}$ pump (Fig. 11(a)), displayed a very fast component with a time constant of few ps and a small amplitude slow decay component with a lifetime of few hundred ps. The fast component was due to electron-phonon coupling, whereas the slower decay was due to phonon relaxation. The excitationtransfer processes including the energy transfer between $\mathrm{CB}$ and internal conversion transition happened at the ps scale and led to the fast decay component in the pump-probe dynamics. The slow decay component corresponded to the evolution of excitation within internal conversion transition states. Upon direct excitation with the laser pump pulse at $325 \mathrm{~nm}$, a negative signal was recorded for the ethanol solution of $\mathrm{Ag}-\mathrm{ZnO}$ nanowires at different wavelengths (Fig. 11(a)). After excitation with $325 \mathrm{~nm}$ pumps and analyses as different wavelengths, the results obtained showed that $\mathrm{Ag}-\mathrm{ZnO}$ materials have the same mechanism derived from ground state bleaching. This sample, when excited using a $380 \mathrm{~nm}$ pump (Fig. 11(b)), displayed the lifetime of the fast component; however, the lifetime varied in the range of 18-36 ps with the increasing probe wavelengths of $480.18,500.41,550.39,600.37$, and $640.83 \mathrm{~nm}$, and a small amplitude slow decay component with a lifetime of $>1.2 \mathrm{~ns}$ was also observed. The fast component was generated due to excited-state to DL coupling, and the slower decay was due to phonon relaxation. The excitation-transfer processes including the nonradiative transition from CB directly to the DL happened in the ps scale, leading to the fast decay component in the pump-probe dynamics. The slow decay component corresponded to the excitation from DL state directly to CB state without internal conversion transition states. Upon direct excitation with the laser pump pulse at $325 \mathrm{~nm}$, a negative signal at different probed wavelengths (Fig. 11(b)) was recorded for the ethanol solution of Ag-ZnO nanowires. After excitation with $380 \mathrm{~nm}$ pumps and analyses with different wavelengths, the results showed that $\mathrm{Ag}-\mathrm{ZnO}$ materials have the same mechanism, which is derived from bleaching of DL. After excitation with a $400 \mathrm{~nm}$ pump (Fig. 11(c)), a very fast component with a time constant of $12-26$ ps and a small amplitude slow decay component with a lifetime of $>1.2 \mathrm{~ns}$ were obtained. The fast component was due to the high excited-state to low excitedstate coupling, whereas the slower decay was due to phonon relaxation. The excitation-transfer processes including the nonradiative decay from the high excited-state directly to a low excited-state led to the fast decay component in the pumpprobe dynamics. The slow decay component corresponded to the evolution of excitation within the internal conversion transition states. Upon direct excitation with the laser pump pulse at $400 \mathrm{~nm}$, a positive signal (Fig. 11(c)) was recorded for the ethanol solution of $\mathrm{Ag}-\mathrm{ZnO}$ nanowires at different probed wavelengths. After excitation with $400 \mathrm{~nm}$ pump and analyses with different wavelengths, the obtained results showed that $\mathrm{Ag}-\mathrm{ZnO}$ exhibits the same mechanism, which is derived from TPA-induced ESA.
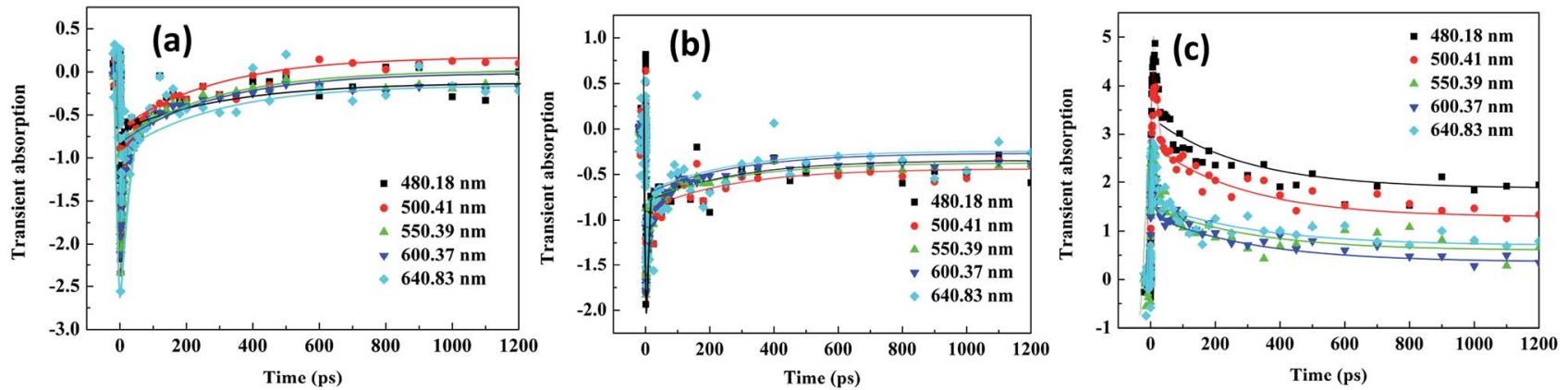

Fig. 11 Pump-probe transients of Ag-ZnO nanowires with sputtering time of 120 s excited at different wavelengths ( 325,380 and $400 \mathrm{~nm}$ ) and probed at different wavelengths. 


\section{Conclusions}

In summary, we have investigated the structural properties, RTPL and LTPL spectra, and the ultrafast nonlinear optical properties and carrier dynamics of the $\mathrm{Ag}-\mathrm{ZnO}$ nanowires. The average diameters of the $\mathrm{Ag}$ nanoparticles synthesized with different sputtering times are nearly the same although their densities are different. Moreover, the RTPL results demonstrate that the samples exhibit stronger visible emission peaks than the unmodified ZnO nanowires. The LTPL spectra show reduced emission, which can be due to re-absorption and scattering of the Ag nanoparticles. The self-focusing effect and nonlinear absorption behavior of $\mathrm{Ag}-\mathrm{ZnO}$ nanowires in ethanol solution have been measured using the $Z$-scan technique. The TPA-induced ground state SA and excited state RSA behavior properties are evident from the experiments using three laser pulses. The ultrafast dynamics process is studied using a TCPP setup with a pulsed laser of $100 \mathrm{fs}$. The results indicate that the carrier dynamics depend strongly on the pump, the probe wavelengths and the bandgap of the material. The experimental results indicate that the few decade picosecond component has been assigned to vibrational relaxation in the excited electronic states, and the slow components represent the decay from $\mathrm{S}_{1}$ to the ground state $(<1.2 \mathrm{~ns})$ and DL to the ground state $(>1.2 \mathrm{~ns})$. These experimental results can be helpful for developing ultrafast optical switching and ultrafast devices for practical applications.

\section{Conflicts of interest}

There are no conflicts to declare.

\section{Acknowledgements}

The National Natural Science Foundation of China (11504072). University Nursing Program for Young Scholars with Creative Talents in Heilongjiang Province (No. UNPYSCT-2016179).

\section{References}

1 D. Panda and T. Y. Tseng, J. Mater. Sci., 2013, 48, 6849-6877. 2 Z. Li, G. Z. Wang, Q. H. Yang, Z. B. Shao and Y. Wang, Nanoscale Res. Lett., 2012, 7, 316.

3 K. P. Jayadevan and T. Y. Tseng, J. Nanosci. Nanotechnol., 2005, 5, 1768.

4 T. B. Guo, Y. Q. Chen, L. Z. Liu, Y. F. Cheng, X. H. Zhang, Q. Li, M. Q. Wei and B. J. Ma, J. Power Sources, 2012, 201, 408-412.

5 S. M. Wang, Z. F. Li, P. Wang, C. H. Xiao, R. Zhao, B. X. Xiao, T. Y. Yang and M. Z. Zhang, CrystEngComm, 2014, 16, 57165723.

6 J. Q. Liu, C. X. Guo, C. M. Li, Y. Y. Li, Q. B. Chi, X. T. Huang, L. Liao and T. Yu, Electrochem. Commun., 2009, 11, 202-205.

7 P. Rauwel, M. Salumaa, A. Aasna, A. Galeckas and E. Rauwel, J. Nanomater., 2016, 2016, 19.

8 E. Rauwel, A. Galeckas, M. R. Soares and P. Rauwel, J. Phys. Chem. C, 2017, 121, 14879-14887.
9 R. S. Devan, R. A. Patil, J. H. Lin and Y. R. Ma, Adv. Funct. Mater., 2012, 22, 3326-3370.

10 Y. N. Xia, P. D. Yang, Y. G. Sun, Y. Y. Wu, B. Mayers, B. Gates, Y. D. Yin, F. Kim and H. Q. Yan, Adv. Mater., 2010, 15, 353389.

11 C. L. Hsu and S. J. Chang, Small, 2015, 10, 4562-4585.

12 C. Soci, A. Zhang, B. Xiang, S. A. Dayeh, D. P. R. Aplin, J. Park, X. Y. Bao, Y. H. Lo and D. Wang, Nano Lett., 2007, 7, 1003.

13 R. P. Prasankumar, P. C. Upadhya and A. J. Taylor, Phys. Status Solidi B, 2010, 246, 1973-1995.

14 L. V. Dao, J. Davis, P. Hannaford, Y. H. Cho, M. A. Green and E. C. Cho, Appl. Phys. Lett., 2007, 90, 067401.

15 K. Makhal, S. Arora, P. Kaur, D. Goswami and K. T. Singh, J. Mater. Chem. C, 2016, 4, 9445-9453.

16 M. A. M. Versteegh, T. Kuis, H. T. C. Stoof and J. I. Dijkhuis, Phys. Rev. B: Condens. Matter Mater. Phys., 2011, 84, 44.

17 M. Montazeri, H. E. Jackson, L. M. Smith, J. M. YarrisonRice, J. H. Kang, Q. Gao, H. H. Tan and C. Jagadish, Nano Lett., 2012, 12, 5389.

18 S. Gul, J. K. Cooper, C. Corrado, B. Vollbrecht, F. Bridges, J. H. Guo and J. Z. Zhang, J. Phys. Chem. C, 2011, 115, 20864-20875.

19 B. P. Meh, J. R. Kirschbrown, M. M. Gabriel, R. L. House and J. M. Papanikolas, J. Phys. Chem. B, 2013, 117, 4390-4398.

20 M. Joschko, P. Langlois, E. R. Thoen, E. M. Koontz, E. P. Ippen and L. A. Kolodziejski, Appl. Phys. Lett., 2000, 76, 1383-1385.

21 K. Appavoo, M. Liu and M. Y. Sfeira, Appl. Phys. Lett., 2014, 104, 1424-1447.

22 D. D. Wang, G. Z. Xing, X. Y. Wang, D. M. Yin, M. Zhou, Q. Guo, J. H. Yang, L. L. Yang, J. Cao and Y. S. Yan, Mater. Res. Bull., 2011, 46, 937-940.

23 C. C. Yang, Y. K. Su, M. Y. Chuang, H. C. Yu and C. H. Hsiao, IEEE Trans. Electron Devices, 2015, 62, 2300-2305.

24 A. S. M. I. Uddin and G. S. Chung, Sensors, 2016, 1-4.

25 Y. H. Ko and J. S. Yu, Phys. Status Solidi A, 2012, 209, 297-301.

26 J. X. Zhang and L. D. Zhang, Adv. Opt. Photonics, 2012, 4, 157321.

27 C. Zhang, H. Y. Xu, W. Z. Liu, L. Yang, J. Zhang, L. X. Zhang, J. N. Wang, J. G. Ma and Y. C. Liu, Opt. Express, 2015, 23, 15565.

28 X. Zhang, Y. Liu and Z. Kang, ACS Appl. Mater. Interfaces, 2014, 6, 4480-4489.

29 M. S. Bahae, A. A. Said and E. W. Van Stryland, Opt. Lett., 1989, 14, 955-957.

30 R. Desalvo, M. Sheik-Bahae, A. A. Said, D. J. Hagan and E. W. Van Stryland, Opt. Lett., 1993, 18, 194.

31 C. B. Yao, Y. D. Zhang, H. T. Yin, Q. Y. Meng, C. Q. Yu, J. Li and P. Yuan, Chem. Phys. Lett., 2013, 576, 35-38.

32 C. B. Yao, Y. D. Zhang, J. Li, D. T. Chen, H. T. Yin, C. Q. Yu and P. Yuan, Opt. Mater., 2014, 37, 80-86.

33 J. Takeda, K. Nakajima, S. Kurita, S. Tomimoto, S. Saito and T. Suemoto, Phys. Rev. B: Condens. Matter Mater. Phys., 2000, 62, 10083-10087.

34 B. Li, R. Tong, R. Zhu, F. Meng, H. Tian and S. Qian, J. Phys. Chem. B, 2005, 109, 10705-10710. 
35 L. Su, N. Qin, W. Xie, J. H. Fu and D. H. Bao, J. Appl. Phys., 2014, 116, 561.

36 C. L. Hsu, Y. D. Gao, Y. S. Chen and T. J. Hsueh, ACS Appl. Mater. Interfaces, 2014, 6, 4277-4285.

37 S. C. Lyu, Y. Zhang, H. Ruh, H. J. Lee, H. W. Shim, E. K. Suh and C. J. Lee, Chem. Phys. Lett., 2002, 363, 134-138.

38 A. Amutha, S. Amirthapandian, B. Sundaravel, B. K. Panigrahi, K. Saravanan and P. Thangadurai, J. Appl. Phys., 2016, 120, 205104.

39 H. P. He, H. P. Tang, Z. Z. Ye, L. P. Zhu, B. H. Zhao, L. Wang and X. H. Li, Appl. Phys. Lett., 2007, 90, 353.

40 M. Liu, S. W. Qu, W. W. Yu, S. Y. Bao, C. Y. Ma, Q. Y. Zhang, J. He, J. C. Jiang, E. I. Meletis and C. L. Chen, Appl. Phys. Lett., 2010, 97, 601 .

41 K. Saravanan, B. K. Panigrahi, R. Krishnan and K. G. M. Nair, J. Appl. Phys., 2013, 113, 189.

42 S. Agnihotri, S. Mukherji and S. Mukherji, RSC Adv., 2013, 4, 3974-3983.

43 K. H. Tam, C. K. Cheung, Y. H. Leung, A. B. Djurisić, C. C. Ling, C. D. Beling, S. Fung, W. M. Kwok, W. K. Chan, D. L. Phillips, L. Ding and W. K. Ge, J. Phys. Chem. B, 2006, 110, 20865.

44 H. P. He, Q. Yang, C. Liu, L. W. Sun and Z. Z. Ye, J. Phys. Chem. C, 2011, 115, 58-64.

45 V. A. Fonoberov, K. A. Alim, A. A. Balandin, F. Xiu and J. Liu, Phys. Rev. B: Condens. Matter Mater. Phys., 2006, 73, 165317.

46 A. Chernikov, M. Koch, B. Laumer, T. A. Wassner, M. Eickhoff, S. W. Koch and S. Chatterjee, Appl. Phys. Lett., 2011, 99, 979.

47 S. K. Srivastava, P. Gangopadhyay, S. Amirthapandian, T. N. Sairam, J. Basu, B. K. Panigrahi and K. G. M. Nair, Chem. Phys. Lett., 2014, 607, 100-104.

48 B. Gu, Y. Sun and W. Ji, Opt. Express, 2008, 16, 17745-17751. 49 S. Perumbilavil, K. Sridharan, A. R. Abraham, H. P. Janardhanan, N. Kalarikkal and R. Philip, RSC Adv., 2016, 6, 106754-106761.
50 B. Anand, R. Podila, P. Ayala, L. Oliveira, R. Philip, S. S. S. Sai, A. A. Zakhidov and A. M. Rao, Nanoscale, 2013, 5, 7271-7276.

51 N. K. M. N. Srinivas, S. V. Rao and D. N. Rao, J. Opt. Soc. Am. $B, 2003,20,2470-2479$.

52 A. E. Siegman, Lasers, University Science, Mill Valley, California, 1986, p. 207.

53 M. S. Malcuit, R. W. Boyd, L. W. Hillman, J. Krasinski and C. R. Stroud, J. Opt. Soc. Am. B, 1984, 1, 73-75.

54 M. Samoc, A. Samoc, B. L. Davies, H. Reish and U. Scherf, Opt. Lett., 1998, 23, 1295-1297.

55 C. B. Yao, X. Wen, Q. H. Li, X. Y. Yan, J. Li, K. X. Zhang, W. J. Sun, L. N. Bai and S. B. Yang, Chem. Phys. Lett., 2017, 671, 113-117.

56 F. J. Zhang, W. F. Guo, X. B. Sun, Q. Ren, Y. Gao, H. L. Yang, G. H. Zhang, Y. T. Chow and D. Xu, Laser Phys. Lett., 2010, 4, 230-233.

57 B. S. Kalanoor and P. B. Bisht, Opt. Commun., 2010, 283, 4059-4063.

58 S. Ijaz, A. Mahendru and D. Sanderson, IEEE J. Quantum Electron., 1990, 26, 760-769.

59 M. T. Cisneros, N. Yanagihara, B. G. Rolon, M. A. M. Nava, O. G. I. Manzano, D. A. M. Arrioja, J. S. Mondragon, E. A. Gomez and L. A. A. Cortes, Microelectron. J., 2009, 40, 621-623.

60 R. Sato, M. Ohnuma, K. Oyoshi and Y. Takeda, Phys. Rev. B: Condens. Matter Mater. Phys., 2014, 90, 125417.

61 Y. P. Chan, J. H. Lin, C. C. Hsu and W. F. Hsieh, Opt. Express, 2008, 16, 19900-19908.

62 T. Voss and J. P. R. A. Dev, Phys. Status Solidi B, 2010, 247, 2476-2487.

63 E. Parilov and M. J. Potasek, J. Opt. Soc. Am. B, 2006, 23, 1894-1910.

64 G. H. Fan, Y. H. Han, S. L. Luo, Y. T. Li, S. L. Qu, Q. Wang, R. X. Gao, M. R. Chen and M. Han, Phys. Chem. Chem. Phys., 2016, 18, 8993-9004. 